\title{
Peritoneal Infusion with Cold Saline Decreased Postoperative Intra-abdominal Adhesion Formation: Letter to the Editor
}

\author{
M. M. Binda $\cdot$ R. Corona $\cdot$ J. Verguts $\cdot$ \\ P. R. Koninckx
}

Published online: 31 August 2010

(c) Société Internationale de Chirurgie 2010

We read with interest the article by Fang et al. [1] demonstrating that peritoneal infusion with cold saline for $30 \mathrm{~min}$ after surgery decreased adhesion formation in a mouse model. These results indeed confirm our data demonstrating that, in a laparoscopic mouse model, adhesion formation decreases with hypothermia [2]. The data are, moreover, consistent with our observation in mice that intraperitoneal temperatures higher than $37^{\circ} \mathrm{C}$ increase adhesion formation [3], as had already been observed after irrigation with saline warmer than $37^{\circ} \mathrm{C}$ [4].

We would be most interested to have some additional information. From the article by Fang et al., it is unclear what the impact of cold saline infusion was upon body temperature of the mice; i.e., to what extent the infusion with cold saline affects the superficial layers, the abdominal cavity, or the entire body. We also were intrigued by the results of cytokines which are nice, but difficult to judge without standard deviations. Moreover we were surprised that some effects lasted for more than 7 days, although it is generally believed that peritoneal repair is completed by postoperative day 7. Also group III is intriguing. Because we know that desiccation occurs when the abdomen is left open, this can be assumed to be the driving mechanism of the increase of adhesions and of the changes in cytokines observed. Therefore, we wonder whether the authors observed any changes in the mesothelium indicating desiccation. Their data do, however, confirm that the peritoneal cavity is a cofactor in adhesion formation at the level of the surgical trauma [5].
Surprisingly, also in open surgery, Fang et al. did not see de novo adhesions, thus confirming our observations in a laparoscopic mouse model. The time courses of the cytokine concentrations in group IV (desiccation only; no surgical trauma) and group III (desiccation + surgical trauma) are intriguing in that opening the abdomen (and desiccation) induced such an increase in adhesions, yet affected cytokines only slightly.

In conclusion, we appreciate the promising results that expand to open surgery our observations that in laparoscopy temperature affects adhesion formation.

\section{References}

1. Fang CC, Chou TH, Lin GS et al (2010) Peritoneal infusion with cold saline decreased postoperative intra-abdominal adhesion formation. World J Surg 34:721-727

2. Binda MM, Molinas CR, Mailova K et al (2004) Effect of temperature upon adhesion formation in a laparoscopic mouse model. Hum Reprod 19:2626-2632

3. Binda MM, Molinas CR, Hansen P et al (2006) Effect of desiccation and temperature during laparoscopy on adhesion formation in mice. Fertil Steril 86:166-175

4. Kappas AM, Fatouros M, Papadimitriou K et al (1988) Effect of intraperitoneal saline irrigation at different temperatures on adhesion formation. Br J Surg 75:854-856

5. Schonman R, Corona R, Bastidas A et al (2009) Effect of upper abdomen tissue manipulation on adhesion formation between injured areas in a laparoscopic mouse model. J Minim Invasive Gynecol 16:307-312
M. M. Binda $(\bowtie) \cdot$ R. Corona · J. Verguts · P. R. Koninckx Department of Obstetrics and Gynaecology, University Hospital Gasthuisberg, Katholieke Universiteit Leuven, Herestraat 49, 3000 Leuven, Belgium

e-mail: Mercedes.Binda@gmail.com 